\title{
Cyclooxygenase-2 in oligodendroglioma: Possible prognostic significance
}

\author{
Anna Maria Buccoliero, ${ }^{1}$ Adele Caldarella, ${ }^{1}$ Luisa Arganini, ${ }^{1}$ Pasquale Mennonna, ${ }^{2}$ Pasquale Gallina, ${ }^{3}$ \\ Antonio Taddei ${ }^{4}$ and Gian Luigi Taddei ${ }^{1}$ \\ ${ }^{1}$ Department of Human Pathology and Oncology, Medical School, University of Florence, ${ }^{2}$ Unit of Neurosurgery, Careggi \\ Hospital, and Departments of ${ }^{3}$ Neurosurgery and ${ }^{4}$ General Surgery, Medical School, University of Florence, Florence, Italy
}

Cyclooxygenase-2 (COX-2) is the inducible form of the enzyme involved in the first two steps of the prostaglandins and thromboxane synthesis. Up-regulation of COX-2 is demonstrated in tumors where it can modulate tumoral progression, metastasis, multidrug resistance and angiogenesis. Selective COX-2 inhibitors are seen with growing interest in the tumors treatment. This present study reviews the COX-2 expression in 32 primary oligodendrogliomas (24 WHO II; eight WHO III) and two glioblastomas with prominent oligodendroglial features (WHO IV). Immunohistochemical results were compared with survival in order to verify the COX-2 prognostic significance. COX-2 positivity was found in $44 \%$ tumors. Median survival of the patients with a COX-2 positive lesion was 37 months; median survival of the patients with a COX-2 negative lesion was 93 months $(P=\mathbf{0 . 0 1 0})$. Twenty-nine percent WHO grade II tumors, 87\% WHO grade III, $50 \%$ WHO grade IV resulted COX-2 positive $(P=0.016)$. In patients affected by WHO grade II oligodendroglioma, median survival was 24 and 96 months, respectively, in COX-2 positive and negative lesions $(P=\mathbf{0 . 0 1 2})$. In conclusion, even if further studies on different, homogeneous and larger series in vivo are certainly necessary, it is believed that $\mathrm{COX}-2$ could really have a prognostic value and can be considered as a possible therapeutic opportunity.

Key words: cyclooxygenase-2, glioma, immunohistochemistry, non-steroidal anti-inflammatory drugs, oligodendroglioma, prognostic factor.

Correspondence: Anna Maria Buccoliero, MD, Department of Human Pathology and Oncology, Medical School, University of Florence, Viale G.B. Morgagni, 85, 50134 Florence, Italy. Email: ambuccoliero@unifi.it

Received 18 November 2003; revised and accepted 26 December 2003.

\section{INTRODUCTION}

Cyclooxygenase (COX) is a membrane-bound enzyme involved in the first two steps of the prostaglandins (PG) and thromboxane (TX) synthesis from arachidonic acid. The existence of at least two COX isoforms designated as COX-1 and COX-2, encoded by separate genes, and catalyzing the same reactions, have been demonstrated. While COX-1 is constitutively expressed almost ubiquitously in mammalian tissues, COX-2 is induced by several stimuli (i.e. proinflammatory signals, hormones, growth factors) and by certain mutations (i.e. v-src, Ha-ras, HER-2/neu, Wnt). ${ }^{1-5}$ However, it has been recently documented that COX-1 can be induced by VEGF, arachidonic acid, forskolin, dibutyryl-cAMP, and $\mathrm{PGE}_{2}$ and that COX-2 can be constitutionally expressed in some organs (i.e. brain and kidney). ${ }^{6-9}$

Lately, another isoform known as COX-3 has been demonstrated and it is especially abundant in the cerebral cortex and in the heart. ${ }^{10}$

Numerous studies showed that COX-2 plays a critical role in the development of neoplasms through various and not completely clear ways. Increased levels of the COX enzyme products are present in the neoplastic tissue, where they can stimulate gene transcription, tumoral growth, angiogenesis, metastasis and immunosoppression, inhibit apoptosis, and cause resistance to chemotherapy through P-glicoprotein-170 over expression.,11-17 Experimental data suggest a possible therapeutic use of the COXinhibitors. Non-steroidal anti-inflammatory drugs (NSAID) can block tumor growth through many mechanisms, especially through antiangiogenic and proapoptotic effects. ${ }^{18,19}$ Moreover, NSAID can also improve the efficacy of radiotherapy, chemotherapy and hormonal therapy. ${ }^{18}$ However, prolonged administration of NSAID can be problematic because of the toxic effects on the gastrointestinal apparatus and on the kidney related to the inhibition 
of COX-1. For this reason, there is growing interest in COX-2-selective inhibitors.

Oligodendroglioma is the third most common glial tumor representing between $5 \%$ and $18 \%$ of all intracranial gliomas. ${ }^{20}$ It principally affects the white matter in the frontal lobe where it is supposed to derive from oligodendrocytes or from a precursor cell committed to oligodendroglial differentiation. ${ }^{20,21}$ Although oligodendroglioma is commonly considered a slow growing chemo-sensible tumor, it can have a worse outcome locally recurring or incurring malignant transformation. ${ }^{20}$

The present study retrospectively reviews the immunohistochemical COX-2 expression of 32 consecutive primary oligodendroglioma and two glioblastomas with prominent oligodendroglial features in which clinical data were available. Immunohistochemical results were compared with survival in order to verify the prognostic significance.

\section{PATIENTS AND METHODS}

\section{Patients}

Thirty-four consecutive patients affected by oligodendroglioma $(32 ; 94 \%)$ or by glioblastoma with prominent oligodendroglial features $(2 ; 6 \%)$ surgically treated at the Neurosurgical Service (Careggi Hospital, Florence, Italy) and at the Department of Neurosurgery (University of Florence, Florence, Italy) between January 1980 and December 2001, were studied.

Fifteen (44\%) were men and 19 (56\%) were women. Average age at the time of the surgery was 53 years (range 30-78 years).

The majority of the tumors ( 14 cases, $41 \%$ ) were localized in the frontal lobe; 1 lesion $(3 \%)$ took origin at the medullary conus level (Table 1: patient no. 2).

Resection was considered macroscopically and radiologically complete in 23 cases $(68 \%)$ and incomplete in six $(18 \%)$. In the remaining five cases $(15 \%)$ the data on the surgical completeness were lacking. In all patients surgery has been performed before any additional treatment.

Twelve patients ( $35 \%$ ) were reoperated on because of a recurrence. In five, a second recurrence was diagnosed.

Fourteen patients $(41 \%)$ were treated with nitrosoureabased chemotherapy (2-31 oral administrations, on average 10 , every 2 months). In 12 cases the chemotherapy followed the excision of the primary lesion whereas, in the remaining two cases it followed the surgical treatment of the recurrence (Table 1: patients no. 13 and 27). Three patients $(9 \%)$, one of these after the resection of the recurrence (Table 1: patient no. 18), underwent radiotherapy.
The histological diagnosis was executed at the Department of Human Pathology and Oncology (University of Florence, Florence, Italy). Twenty-six oligodendrogliomas $(76 \%)$ were pure forms and six (18\%) were mixed oligo-astrocytomas; the remaining two cases $(6 \%)$ were glioblastomas with prominent oligodendroglial features. Twenty-four oligodendrogliomas (71\%) were classified as WHO grade II and eight (24\%) as WHO III. ${ }^{20}$

Median survival (follow-up was available in 29 patients) was 68 months (range 1-193). At the end of the study, 22 patients had died and seven were alive and free of disease at 24, 24, 27, 28, 63, 102 and 193 months. Clinical, histopathological and immunohistochemical data are shown in Table 1.

\section{Immunohistochemical study}

Five- $\mu \mathrm{m}$-thick sections of the most representative specimen of each case were mounted on electrostatic slides for immunohistochemical evaluation. Sections were deparaffined, rehydrated and subjected to antigen retrieval by automated microwaving (MicroMED T/T Megamilestone Srl Sorisole Bergamo, Italy) in TEC (Tris-EDTA-Citrate) buffer ph 7.8 for $36 \mathrm{~min}$. Endogenous peroxidase was blocked with $3 \% \mathrm{H}_{2} \mathrm{O}_{2}$ in distilled water. All tumor specimens were immunostained with primary goat polyclonal antibody anti-COX-2 (1:40 dilution; C-20; Santa Cruz Biotechnology, Santa Cruz, CA, USA) for $2 \mathrm{~h}$ at room temperature. Then, sections were treated with secondary antibody (1:100 dilution; biotinylated rabbit antigoat IgG, Dako Cytomation, Glostrup, Denmark) for $1 \mathrm{~h}$ and with streptavidin horseradish peroxidase (Laboratory Vision, Fremont, CA, USA) for $10 \mathrm{~min}$, developed with 3,3 diaminobenzidine-hydrogen peroxide (BioGenex, San Ramon, CA, USA) and counterstained with hematoxylin.

The following procedures were used as negative controls: (i) a non-immune serum in place of the primary antibody, and (ii) the primary antibody was omitted. An ascertained COX-2 positive colon adenocarcinoma was used as positive control.

COX-2 expression was indicated as ' + ' when it was present on the cytoplasm of more than $25 \%$ of the cells, and as '-' when it was focal or absent.

\section{Statistical analysis}

Survival was calculated from the date of diagnosis to the date of death or last follow-up, and was analyzed by the Kaplan-Meier method. The Log-rank test was used to assess the strength of association between survival time and COX-2 expression.

Statistical significance was defined as $P<0.05$. 


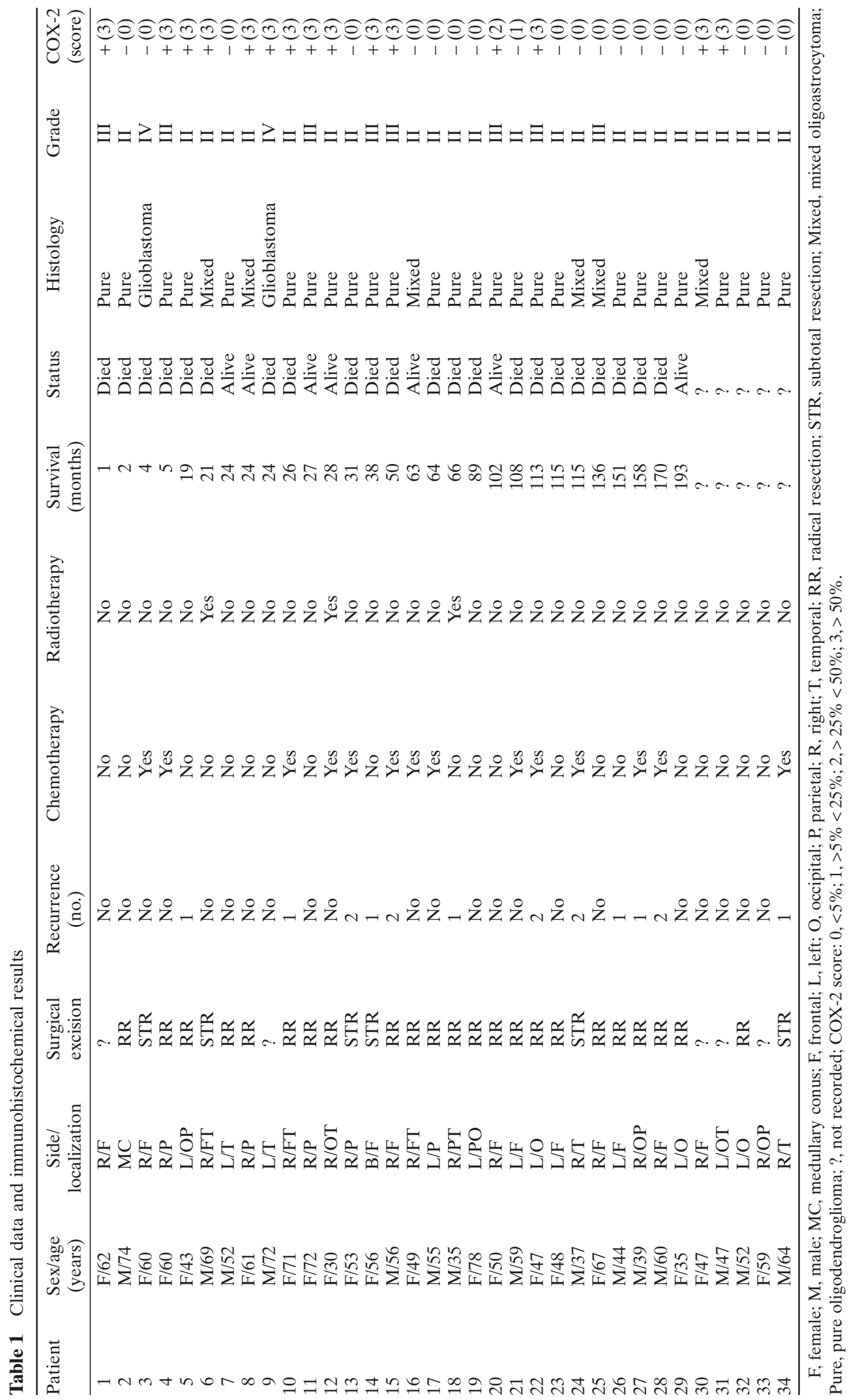




\section{RESULTS}

COX-2 positivity was found in 15 of 34 (44\%) tumors (neoplastic cells, endothelial cells and cells having the morphological features of neurons and astrocytes). In the remaining 19 (56\%) COX-2 negative cases, endothelial and occasional cells having the morphological features of neurons and astrocytes resulted in COX-2 positive (Table 1; Fig. 1).

Follow-up was available in 29 patients (Table 1).

Median survival of the 13 patients with a COX-2 positive lesion was 37 months; median survival of the 16 patients with a COX-2 negative lesion was 93 months $(P=0.010)$ (Fig. 2).

Seven of $24(29 \%)$ WHO grade II oligodendrogliomas, seven of eight (87\%) WHO grade III, and one of two
$(50 \%)$ WHO grade IV resulted in COX-2 positive $(P=0.016)$ (Fig. 3).

Median survival of 19 patients with a WHO grade II oligodendroglioma was 77 months; median survival of the patients with a WHO grade III was 59 months; median survival of the patients with a WHO grade IV was 14 months $(P>0.05 ; \mathrm{ns})$.

In the group of 19 patients affected by WHO grade II oligodendroglioma median survival was 24 and 96 months, respectively, in COX-2 positive and negative lesions $(P=0.012)$ (only one WHO III and one WHO IV lesion were COX-2 negative) (Fig. 4).

Median survival of 13 patients treated with chemotherapy was 72 months; in this group median survival was 44 and 89 months, respectively, in COX-2 positive and negative lesions $(P>0.05$; ns $)$.
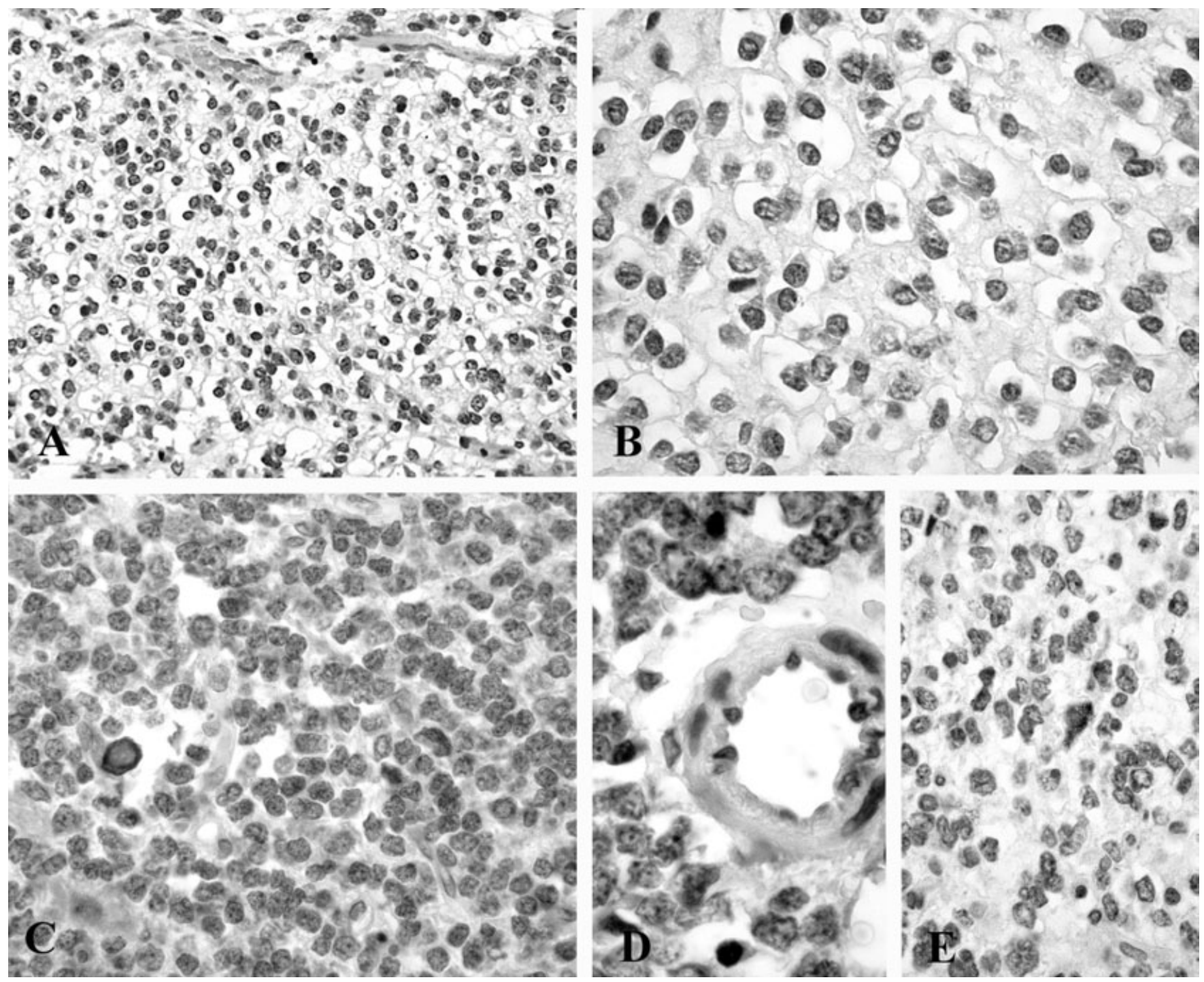

Fig. 1 Cytoplasmic immunostaining in (A-B) WHO II and (C-D) WHO III oligodendrogliomas; (D) positive endothelial cells; (E) COX-2 negative oligodendroglioma with entrapped astrocytes showing positive immunoreaction. 


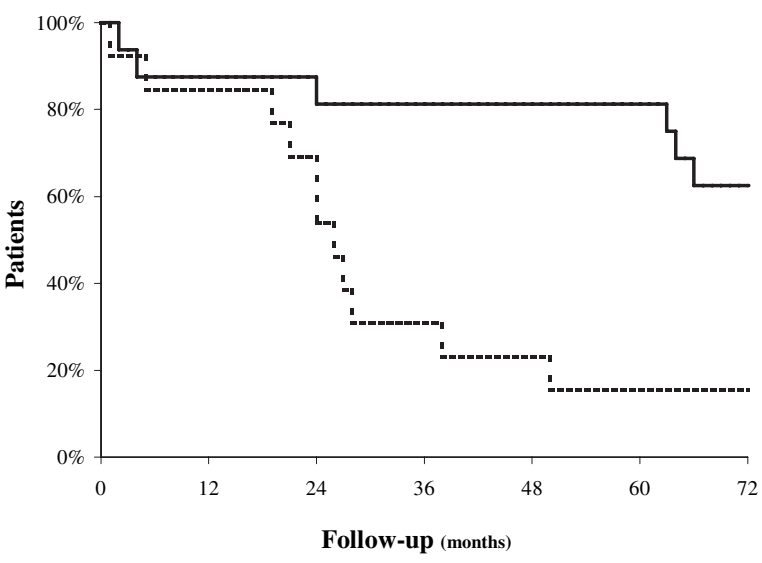

Fig. 2 Median survival of 13 patients with COX-2 positive lesion was 37 months (broken line); median survival of 16 patients with COX-2 negative lesion was 93 months (continuous line) $(P=0.010)$.

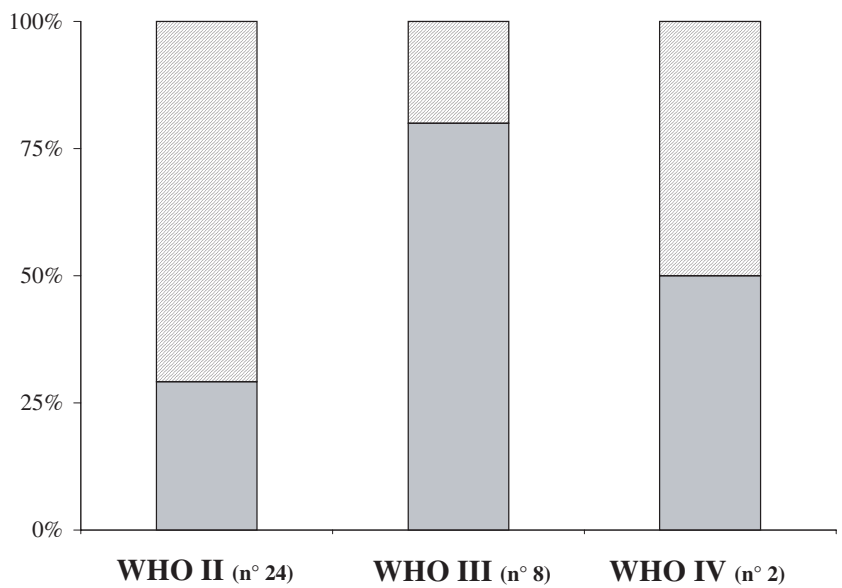

Fig. 3 Seven of 24 (29\%) WHO grade II oligodendrogliomas, seven of eight $(87 \%)$ WHO grade III, and one of two $(50 \%)$ WHO grade IV resulted COX-2 positive (light gray) $(P=0.016)$.

Five of 15 (33\%) COX-2 positive and seven of $19(37 \%)$ COX-2 negative oligodendroglioma relapsed $(P>0.05$; ns).

In the group of patients that were alive at the end of the study, four (57\%), alive at 24, 27, 28, and 102 months, had COX-2 positive lesions; three (43\%), alive at 24,63 , and 193 months, had COX-2 negative lesions.

\section{DISCUSSION}

The role of COX-2 has been studied in many pathologies from arthritis to Alzheimer's disease to cancer. In the neoplastic context, the COX-2 function has been best worked out in colorectal cancer where robust results indicate an important role of this enzyme in the tumoral progression:

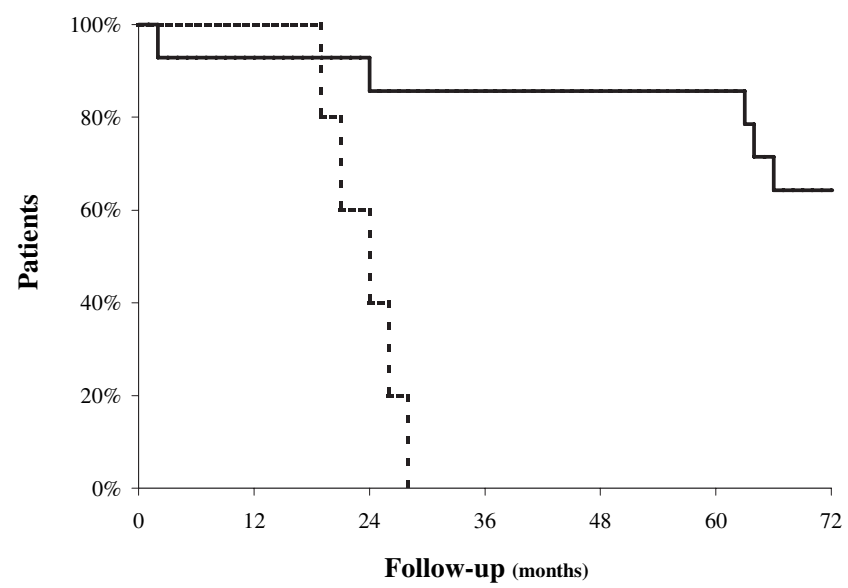

Fig. 4 In patients affected by WHO grade II oligodendroglioma, median survival was 24 and 96 months, respectively, in COX-2 positive (broken line) and negative (continuous line) lesions $(P=0.012)$.

adenomatous polyps and malignant colonic tumors express higher level of COX-2 respect to the normal intestinal mucosa; NSAID significantly reduce the risk of insurgence of colorectal tumor and the number of the pre-existing adenoma in adenomatous familial syndrome affected patients. $^{19,22}$

Several experimental studies show that the expression of COX-2 is also detectable in gliomas, and suggest that it is grade and prognosis related. ${ }^{2,3,14,23-27}$

The most interesting data regard the possibility of a therapeutic use of COX-2 inhibitors in the treatment of gliomas. Already in 1990, it was demonstrated that the inhibitors of eicosanoid biosynthesis suppress proliferation and promote differentiation in glioblastoma. ${ }^{28}$ More recently, the antitumoral effects of SC-236, NS-398 (selective COX-2 inhibitors) and of flurbiprofen were proven., ${ }^{2,29}$

There is a paucity (only two studies) of data about the expression and the specific role of COX-2 in oligodendroglioma. . $3,27^{2}$

In 2000, Deininger et al. first investigated the expression of COX-2 in oligodendrogliomas by immunohistochemistry. ${ }^{23}$ They observed a COX-2 immunoreactivity limited at the astrocytes, neurons and endothelial cells, particularly in areas of infiltrative tumor growth. The number of COX-2 positive cells was significantly lower in primary oligodendrogliomas than in high grade oligodendroglioma relapsed. The authors supposed a cyclooxygenase accumulation during oligodendroglioma progression in vivo.

In 2003, Castilla et al. studied further, immunohistochemically, COX-2 expression in oligodendrogliomas. ${ }^{27}$ They noted a cytoplasmic immunoreactivity in tumoral cells of approximately $18 \%$ of cases studied but, contrary to Deiniger et al., they noted that the reactive astrocytes were COX-2 negative. Castilla et al. concluded that nega- 
tive immunostaining for COX-2 correlated with a higher survival rate.

In both studies a COX-2 influence on the prognosis was suggested even if there were discordant results about the percentage and the prevalent type (astrocytes, neurons and endothelial cells in the first study, neoplastic cells in the second study) of cells expressing COX-2, probably related to the different immunohistochemical methods.

Our present findings documented a frequent (44\%) expression of COX-2 (Fig. 1) in oligodendroglial neoplasms (neoplastic cells, entrapped neurons and astrocytes, and endothelial cells) and gave ulterior support to the hypothesis that $\mathrm{COX}-2$ can influence the prognosis. In particular, patients with a COX-2 negative lesion survived longer than patients with a COX-2 positive lesion when considering all patients (37 vs 93 months; $P=0.010$; Fig. 2) and also when studying the WHO II oligodendrogliomas separately (24 vs 96 months; $P=0.012$; Fig. 4) (follow-up occurred for 29 patients). Furthermore, COX-2 resulted in less expression in low-grade than high-grade lesions $(29 \%$ in WHO grade II, $87 \%$ in WHO grade III, and $50 \%$ in WHO grade IV lesions; $P=0.016$; Fig. 3 ).

The shown capacity of COX-2 products to stimulate tumoral growth and progression can explain the poor prognosis of COX-2 positive lesions and the frequent COX-2 expression in high-grade oligodendrogliomas.

In spite of recent data suggesting a possible COX-2 related chemo resistance, ${ }^{13,15,16}$ we ascertained that the COX-2 expression does not seem to modify the survival time in oligodendroglioma affected patients treated with chemotherapeutic agents (median survival of the patients treated with chemotherapy was 44 months when COX-2 was positive, and 89 months when $\mathrm{COX}-2$ was negative; $P>0.05)$.

In conclusion, although further studies on different, homogeneous and larger series in vivo are certainly necessary, we believe that COX-2 might really have a prognostic value and could be considered as a possible therapeutic opportunity.

\section{REFERENCES}

1. Daniel TO, Liu H, Morrow JD, Crews BC, Marnett LJ. Thromboxane $\mathrm{A}_{2}$ is a mediator of cyclooxygenase-2dependent endothelial migration and angiogenesis. Cancer Res 1999; 59: 4574-4577.

2. Petersen C, Petersen S, Milas L, Lang FF, Tofilon PJ. Enhancement of intrinsic tumor cell radiosensitivity induced by a selective cyclooxygenase- 2 inhibitor. Clin Cancer Res 2000; 6: 2513-2520.

3. Shono H, Tofilon PJ, Bruner JM, Owalabi O, Lang FF. Cyclooxygenase-2 expression in human gliomas: prog- nostic significance and molecular correlations. Cancer Res 2001; 61: 4375-4381.

4. Vadlamudi R, Mandal M, Adam L, Steinbach G, Mendelson J, Kumar R. Regulation of the cycooxygenase2 pathway by HER2 receptor. Oncogene 1999; 18: 305314.

5. Araki Y, Okamura S, Hussain SP et al. Regulation of cyclooxygenase-2 expression by the Wnt and ras pathway. Cancer Res 2003; 63: 728-734.

6. Bryant CE, Appleton I, Mitchell JA. Vascular endothelial growth factor up-regulates constitutive cyclooxygenase-1 in primary bovine and human endothelial cells. Life Sci 1998; 62: 2195-2201.

7. Maldve RE, Kim Y, Muga SJ, Fischer SM. Prostaglandin $E_{2}$ regulation of cyclooxygenase expression in keratinocytes is mediated via cyclic nucleotidelinked prostaglandin receptors. J Lipid Res 2000; 41: 873-881.

8. Otto JC, Smith WL. Prostaglandin endoperoxide synthases-1 and -2. J Lipid Med Signal 1995; 12: 139156.

9. Herschman HR. Prostaglandin synthase-2. Biochim Biophys Acta 1996; 1299: 125-140.

10. Chandrasekharan NV, Dai H, Roos KL et al. COX-3, a cyclooxygenase-1 variant inhibited by acetaminophen and other analgesic/antipyretic drugs. cloning structure, and expression. Proc Natl Acad Sci USA 2002; 99: 13926-13931.

11. Castelli MG, Chiabrando C, Fanelli R et al. Prostaglandin and tromboxane synthesis by human intracranial tumors. Cancer Res 1989; 49: 1505-1508.

12. Paoletti P, Chiabrando C, Gaetani P et al. Prostaglandins in human brain tumors. J Neurosurg Sci 1989; 33: 65-69.

13. Fantappiè O, Masini E, Sardi I et al. The MDR phenotype is associated with the expression of COX-2 and iNOS in a human hepatocellular carcinoma cell line. Hepatology 2002; 35: 843-852.

14. King JG, Khalili K. Inhibition of human brain tumor cell growth by the anti-inflammatory drug, flurbiprofen. Oncogene 2001; 20: 6864-6870.

15. Patel VA, Dunn MJ. Sorokin a Regulation of MDR-1 (P-glycoprotein) by cyclooxygenase-2. J Biol Chem 2002; 41: 38915-38920.

16. Ratnasinghe D, Dascher PJ, Anver MR et al. Cyclooxygenase-2, P-glycoprotein-170 and drug resistance; is chemoprevention against multidrug resisrance possible? Anticancer Res 2001; 21: 21412147.

17. Sheng H, Shao J, Morrow JD, Beauchamp D, DuBois RN. Modulation of apoptosis and Bcl-2 expression by prostaglandin $\mathrm{E}_{2}$ in human colon cancer cells. Cancer Res 1998; 58: 362-366. 
18. Gasparini G, Longo R, Sarmento R, Morabito A. Inhibitors of cyclo-oxygenase 2: a new class of anticancer agents? Lancet Oncol 2003; 4: 605-615.

19. Haller DG. COX-2 inhibitors in oncology. Semin Oncol 2003; 30: 2-8.

20. Kleihues P, Cavenee WK. Pathology and Genetics of Tumours of the Nervous System. Lyon: IARC Pres, 2000.

21. Schiffer D. Brain Tumors. Biology, Pathology and Clinical References. Berlin: Lüderitz \& Bauer, 1997.

22. Einspahr JG, Krouse RS, Yochim JM et al. Association between Cyclooxygenase expression and colorectal adenoma characteristics. Cancer Res 2003; 63: 38913893.

23. Deininger $\mathrm{MH}$, Meyermann $\mathrm{R}$, Trautmann $\mathrm{K}$ et al. Cyclooxygenase (COX)-1 expressing macrophages/ microglial cells and COX-2 expressing astrocytes accumulate during oligodendroglioma progression. Brain Res 2000; 885: 111-116.

24. Deininger MH, Weller M, Streffer J, Mitterbronn M, Meyermann R. Patterns of cyclooxygenase-1 and -2 expression in human gliomas in vivo. Acta Neuropathol 1999; 98: 240-244.

25. Kurzel F, Hagel CH, Meissner H, Westphal M, Giese A. Cyclo-oxygenase inhibitors and tromboxane synthase inhibitors differentially regulate migration arrest, growth inhibition and apoptosis in human glioma. Acta Neurochir (Wien) 2002; 144: 71-87.

26. Prayson RA, Castilla EA, Vogelbaum MA, Barnett GH. Cyclooxygenase-2 (COX-2) expression by immunohistochemistry in glioblastoma multiforme. Ann Diagn Pathol 2002; 6: 148-153.

27. Castilla EA, Prayson RA, Kanner AA et al. Cyclooxygenase-2 in oligodendroglial neoplasms. Cancer 2003; 98: $1465-1472$.

28. Wilson DE, Anderson KM, Seed TM. Ultrastructural evidence for differentiation in a human glioblastoma cell line treated with inhibitors of eicosanoid metabolism. Neurosurgery 1990; 27: 523-531.

29. Joki T, Heese O, Nikas DC et al. Expression of cyclooxygenase 2 (COX-2) in human glioma and in vitro inhibition by a specific COX-2 inhibitor, NS-398. Cancer Res 2000; 60: 4926-4931. 